\title{
METÁSTASIS RETRORBITARIA COMO MANIFESTACIÓN INICIAL DEL CÁNCER DE PRÓSTATA
}

\author{
M.A. BONILLO GARCÍA, J.A. QUEIPO ZARAGOZÁ, J.A. PALMERO MARTI, \\ J.J. PACHECO BRU, F.J. VERA SEMPERE*, J.F. JIMÉNEZ CRUZ \\ Servicio de Urología. *Servicio de Anatomía Patológica. Hospital Universitario La Fe de Valencia.
}

Actas Urol Esp. 27 (7): 562-565, 2003

\section{RESUMEN}

METÁSTASIS RETRORBITARIA COMO MANIFESTACIÓN INICIAL DEL CÁNCER DE PRÓSTATA

La metástasis orbitaria es una localización infrecuente dentro de la diseminación tumoral del cáncer de próstata. Del mismo modo, resulta excepcional que ésta sea la responsable de las manifestaciones clínicas que condicionan el diagnóstico inicial de dicha neoplasia.

Presentamos el caso de un paciente con adenocarcinoma de próstata que debutó con alteraciones de la sensibilidad facial y exoftalmos del ojo derecho. Describimos como se llegó al diagnóstico definitivo y la respuesta al tratamiento hormonal supresivo. La supervivencia del paciente ha sido superior al de la mayoría de casos documentados, superando los 30 meses de seguimiento.

PALABRAS CLAVE: Metástasis retrorbitaria. Adenocarcinoma de próstata. Exoftalmos.

\section{ABSTRACT}

RETRORBITAL METASTASIS AS INITIAL MANIFESTATION OF PROSTATIC CANCER

Orbital metastasis is an unusual localization within tumoral dissemination of prostatic cancer. Similarly, it is rare that orbital metastasis might be responsible for the clinic manifestations that determine the initial diagnosis of neoplasia.

We illustrate the case of a patient suffering from prostatic adernocarcinoma that displayed alterations of facial sensitivity and right eye exophthalmos. We describe how the final diagnosis was reached and the patient's response to the suppressive hormonal treatment. The patient's rate of survival has proved to be longer than the rest of cases documented, with over 30-month follow-up.

KEY WORDS: Retrorbitarial metastasis. Prostatic adenocarcinoma. Exophthalmos.

$\mathrm{E}_{\mathrm{n}}^{1}$ adenocarcinoma de próstata (ADCP) es la neoplasia urogenital más frecuente, supone en la actualidad la tercera causa de muerte por cáncer en el varón tras el pulmonar y el colorrectal. Dado que los métodos de screening (tacto rectal y PSA) no son lo suficientemente sensibles para su diagnóstico precoz, entre un $20-30 \%$ de los pacientes diagnosticados de novo presentan tumores localmente avanzados o enfermedad metastásica ${ }^{1-3}$.

Las localizaciones metastásicas del ADCP más frecuentes son los ganglios linfáticos pélvicos y el esqueleto óseo axial. La metástasis intracraneal es inusual, y cuando ésta tiene lugar general- mente ha sido precedida del diagnóstico de carcinoma de próstata y la enfermedad se ha diseminado por el resto del organismo ${ }^{4}$.

Las metástasis representan entre el 2-10\% de todas las neoplasias orbitarias del adulto. Determinados autores estiman que alrededor de un $30 \%$ de las metástasis orbitarias son la manifestación inicial de neoplasias primarias ocultas ${ }^{5}$. La mayoría de estas metástasis proceden de neoplasias de mama y pulmón (sin considerar leucemias ni linfomas) $)^{6,7}$; no obstante, las de origen prostático, aunque excepcionales, constituyen la segunda causa más frecuente en el hombre, siendo la mayoría detectadas en autopsias ${ }^{3,8}$. 


\section{CASO CLÍNICO}

Presentamos el caso de un varón de 66 años cuyos antecedentes más relevantes son un estrabismo congénito del ojo izquierdo, una dislipemia sin tratamiento médico, una psoriasis y una diabetes mellitus tipo 2 de más de 10 años de evolución mal controlada mediante antidiabéticos orales (Glibenclamida).

En septiembre de 1999 acude a Urgencias de nuestro hospital por dolor supraciliar derecho continuo e intenso de 15 días de evolución, el cual había ido aumentando en extensión e intensidad, asociado a un breve episodio aislado de pérdida de agudeza visual y a una sensación disestésica en la región frontal. Ante esta sintomatología es valorado inicialmente por los servicios de Neurología y Oftalmología, describiendo una hiperestesia en la región de la primera rama del trigémino derecha y un exoftalmos no doloroso del ojo derecho. Se determina una presión intraocular de $14 \mathrm{mmHg}$, una correcta reactividad pupilar $(3 \mathrm{~mm})$ y en el fondo de ojo un exudado blanquecino en el territorio de la arteria temporal izquierda con el borde nasal de la papila borrado junto a pequeñas hemorragias en llama. Una vez ingresado, se solicita una RMN para establecer el diagnóstico diferencial entre una mucormicosis retrorbitaria y una posible infiltración neoplásica de los senos frontal y esfenoidal derechos.

Durante su estancia, aqueja molestias hipogástricas y disminución de la diuresis en las últimas horas, por lo que avisan al servicio de Urología que, tras confirmar la presencia de un cuadro de retención aguda de orina, realiza un sondaje vesical obteniendo $800 \mathrm{ml}$ de orina clara. Mediante una anamnesis dirigida, se descubren alteraciones en la micción en los últimos meses (polaquiuria, nicturia y urgencia) sin hematuria. Al realizar un tacto rectal se aprecia una próstata de gran tamaño (III/IV) fija, no dolorosa, de consistencia pétrea, lo que determina la sospecha clínica de la presencia de un cáncer de próstata. Ante este hallazgo se determina una analítica que incluye PSA y una biopsia prostática ecodirigida.

En la analítica sanguínea obtenemos un PSA de $1450 \mathrm{ng} / \mathrm{ml}$, unas fosfatasas alcalinas de 658 U/L y una velocidad de sedimentación de 57. En los estudios de imagen (RMN y TAC) (Figs. 1 y 2) se advierte un seno etmoidal derecho ocupado y abombado que se biopsia, revelando la presencia de metástasis de un adenocarcinoma positivo en la inmunohistoquímia al PSA, y por tanto compatible con un origen prostático (Fig. 3). El informe anatomopatológico de la biopsia de próstata con-

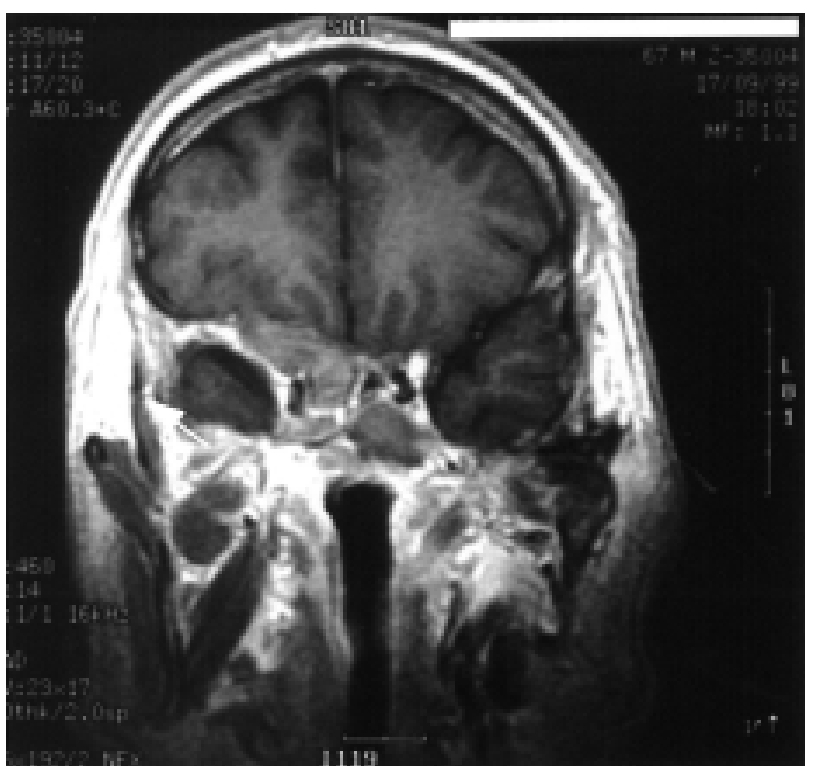

FIGURA 1. RMN coronal donde se aprecia una ocupación parcial del seno esfenoidal derecho y de las celdillas etmoidales posteriores derechas (1999).

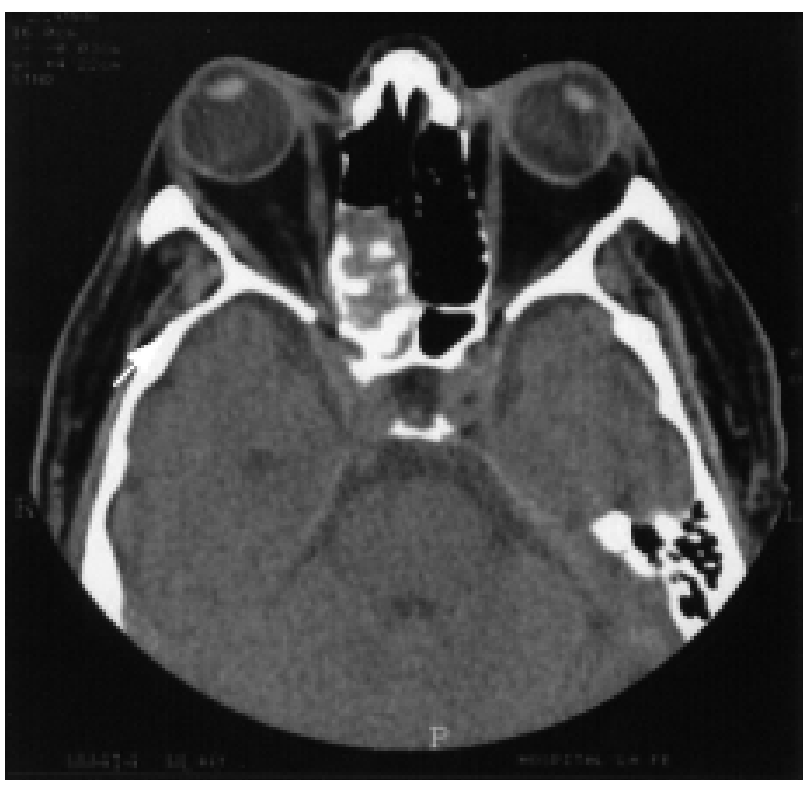

FIGURA 2. TAC de senos en la que se observa ocupación de las celdillas etmoidales posteriores derechas y seno esfenoidal derecho por masa de partes blandas que origina esclerosis del hueso de las paredes (1999). 
firma la presencia de $\mathrm{ADCP}$ en las muestras remitidas (6) con un Gleason de 3+3. Se realiza un estudio de extensión mediante rastreo óseo, descubriendo múltiples acúmulos patológicos de actividad diseminados por todo el esqueleto que sugieren extensa infiltración tumoral (Fig. 4).

Instauramos terapia hormonal supresiva en septiembre de 1999 mejorando la sintomatología neurológica a los 3 meses de iniciado el tratamiento. Se realiza un seguimiento durante 30 meses en el cual el paciente se mantiene asintomático durante el primer año. Analíticamente se

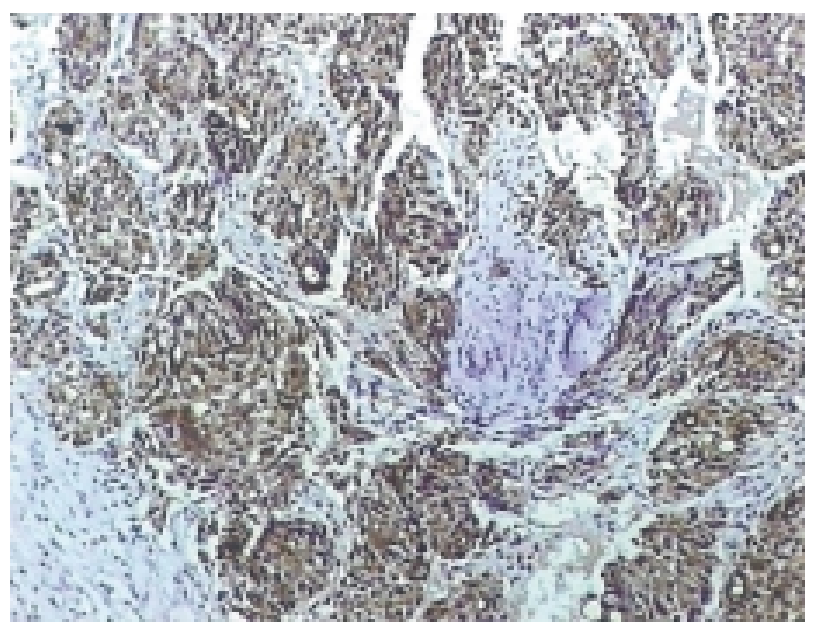

FIGURA 3. Imagen panorámica de exéresis biópsica de lesión etmoidal derecha, con reactividad inmunohistoquimica frente al PSA.

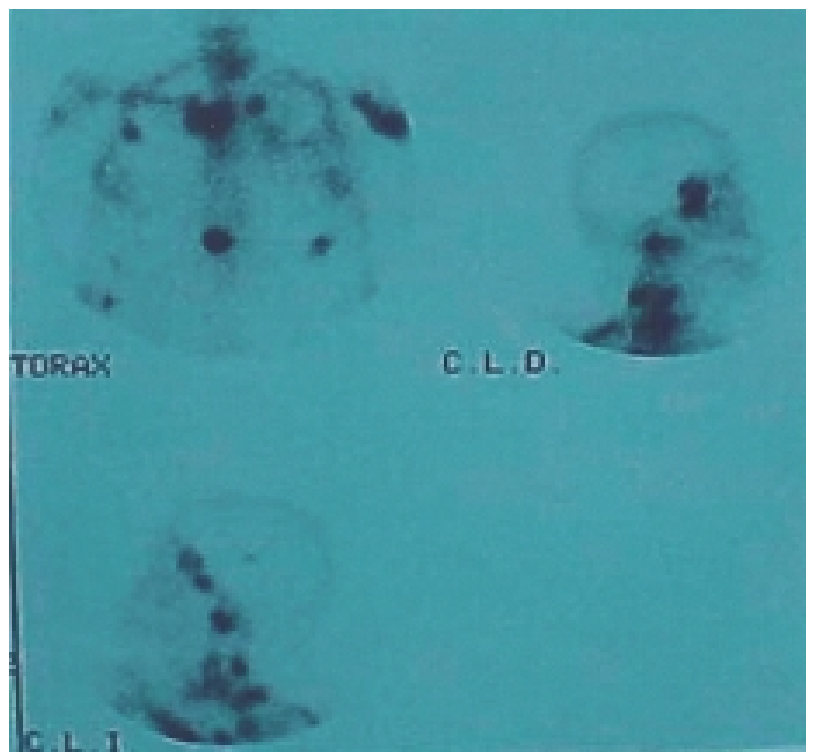

FIGURA 4. Rastreo óseo con Tcm99+, múltiples acúmulos patológicos diseminados, que sugieren infiltración tumoral. Detalle de acúmulo en órbita derecha (1999). aprecia una disminución del PSA de hasta 8,2 $\mathrm{ng} / \mathrm{ml}$ a los 5 meses del diagnóstico, pero con rápido ascenso llegando a un PSA de $83 \mathrm{ng} / \mathrm{ml} \mathrm{a}$ los 14 meses, por lo que se lleva a cabo en este momento un bloqueo hormonal completo. En el rastreo óseo practicado a los 24 meses, se aprecia la desaparición del acúmulo anómalo de actividad intracraneal, aunque persiste la infiltración metastásica del resto del esqueleto, hallazgos que se corroboraron mediante la TAC.

El paciente sufre un empeoramiento progresivo de su estado general, así como pérdida de la agudeza visual. Igualmente, se hace evidente un agravamiento de la sintomatología miccional obstructiva (flujos máximos de $5,7 \mathrm{ml} / \mathrm{s}$, con residuos de $100 \mathrm{ml}$ ). El paciente es controlado por sus algias en la Unidad del Dolor, no acudiendo al último control de Urología por situación terminal en marzo de 2002, 30 meses después de su diagnóstico.

\section{DISCUSIÓN}

El adenocarcinoma de próstata es una neoplasia con un lento patrón evolutivo, su curso clínico es silente, manifestándose generalmente como una uropatía obstructiva. No obstante, algunos autores estiman en un 10-20\% el porcentaje de neoplasias de próstata diagnosticadas por manifestaciones secundarias a su diseminación metastásica ${ }^{3}$. En la bibliografía revisada, tanto inglesa como en castellano, de casos documentados de metástasis orbitarias en el cáncer de próstata, sólo 18 constituyen el debut de la enfermedad neoplásica.

La vía de diseminación suele ser linfática y hematológica. Esta última es fundamentalmente venosa a través del plexo intervertebral de Batson, lo que explicaría que la afectación ósea sea predominantemente axial. De igual modo, justificaría la diseminación intracraneal hacia las leptomeninges, la cual constituye la localización metastásica intracraneal más frecuente. Sin embargo, las metástasis orbitarias necesariamente se producen por vía arterial ${ }^{3}$, mediante émbolos tumorales que sobrepasan el filtro pulmonar.

La sintomatología neurológica, proptosis, diplopia, dolor, disminución de la agudeza visual, etc., al igual que la rápida instauración de la 
misma, la comparte con otros procesos expansivos intracraneales con los que hay que establecer un diagnóstico diferencial. Para delimitar el origen de las manifestaciones clínicas solicitamos inicialmente distintas técnicas de neuroimagen. No obstante, estas exploraciones también podrían conducir a un diagnóstico inicial erróneo, dado que las características radiológicas de un meningioma en placa, por ejemplo, y una metástasis retrorbitaria de un ADCP son similares, presentando ambas un patrón osteoblástico. Por lo tanto, la técnica que con más fiabilidad puede determinar el origen prostático de una masa intracraneal, etmoidal en nuestro caso, será la biopsia y su confirmación histológica mediante la positividad inmunohistoquímica al antígeno prostático específico (PSA). A pesar de esto, se cuestiona su realización en aquellos pacientes en los cuales está confirmada la existencia de un cáncer de próstata metastásico, quedando relegada a aquellos casos cuyo diagnóstico es incierto.

En lo que respecta al tratamiento de elección, la decisión estará determinada por el estado evolutivo de la enfermedad y las condiciones generales del paciente. Dado el bajo número de pacientes, no se han podido establecer estudios concluyentes acerca de la eficacia de los distintos tratamientos paliativos, sin embargo, la instauración precoz del tratamiento parece ofrecer un mejor control sintomático ${ }^{1}$. La radioterapia externa, aislada o combinada con la cirugía, ha sido eficaz en aquellos pacientes donde la metástasis lleva poco tiempo de evolución y la enfermedad no está diseminada ${ }^{1} \mathrm{y}$ al igual que el tratamiento hormonal supresivo, ha mejorado la calidad de vida del enfermo.

En nuestro caso, con el tratamiento médico la sintomatología neurológica mejoró notablemente y se erradicó la masa tumoral retrorbitaria, no siendo necesaria la adyuvancia con radioterapia externa.

La supervivencia de estos pacientes no está correctamente documentada, aunque en la revisión de Olivier y cols. se estima en 16,3 meses, sin encontrar diferencias estadísticamente significativas respecto a otras metástasis del cáncer de próstata. Sorprendentemente Boldt y cols., advierten un mejor pronóstico en aquellos pacientes que debutan con manifestaciones secundarias a la diseminación orbitaria. En nuestro caso, la supervivencia del paciente ha sido superior a 30 meses, por encima de la mayoría de casos similares publicados.

Como conclusión resaltar que el cáncer de próstata debería de ser considerado en el diagnóstico diferencial de masas orbitarias en pacientes de edad avanzada con sintomatología neurológica de reciente aparición y lesiones intracraneales sugestivas, aún cuando no presente sintomatología urológica conocida.

\section{REFERENCIAS}

1. PIERAS E, ROSALES A, LÓPEZ H, VILLAVICENCIO H, VICENTE J.: Metástasis intracraneales en cáncer de próstata. Actas Urol Esp 2000; 24: 626-631.

2. FRANCO E, GIL-NÉCIJA E, CANO G, ESPINOSA R, RUIZ-MATEOS R, OLLERO M.: Metástasis esfenoidal simulando meningioma como manifestación inicial de adenocarcinoma de próstata. Rev Neurol 1999; 29: 929-932.

3. ROMERO P, PELLUCH A, LOBATO JJ y cols.: Exoftalmos por metástasis orbitaria de carcinoma prostático. Esp Urol 1991; 44: 191-194.

4. SUTTON MA, WATKINS HL, GREEN LK, KADMON D.: Intracranial metastases as the first manifestation of prostate cancer. Urology 1996; 48: 789-793.

5. CARRIERE VM, KARCIOGLU ZA, APPLE DJ, INSLER MS.: A case of prostate carcinoma with bilateral orbital metastases and the review of the literature. Ophthalmology 1982; 89: 402-406.

6. PRAT-BARTOMEU J.: Metástasis orbitarias en el adulto. Rev Neurol 2000; 31: 1261-1263.

7. BOLDT HC, NERAD JA.: Orbital metastases from prostate carcinoma. Arch Ophthalmol 1988; 106: 1403-1408.

8. GONZÁLEZ C, KUZEL T, CARTER M.: Metastatic adenocarcinoma of the prostate to the orbit as a presenting symptom. J Urol 1997; 157: 625.

9. AUBERT J, IRANI J, SAINT-BLANCATP.: Orbital metastasis of prostatic cancer. Clinical and therapeutic aspects. A propos of a case Chirurgie 1997; 121: 672-675.

10. OLIVIER C, LAO AH, SALAS C, CARBALLIDO J.: Metástasis de origen prostático en tejidos blandos periorbitarios. Actas Urol Esp 1992; 16: 79-82.

11. SAKAI H, IGAWA T, SAHA PK et al.: A case of prostatic carcinoma presenting as a metastasic orbital tumor. Hinyokika Kiyo 1992; 38: 77-80.

12. RIGOT JM, DURET MH, CASTIER P, MAZEMAN E.: À propos de deux cas de métastases oculo-orbitaires d'un epithélioma prostatique. Ann Urol (París) 1989; 23: 43-45.

13. HARNETT P, RAGHAVAN D, LANGDON P, DUVAL P.: Orbital metastasis from prostatic carcinoma. Br J Urol 1987; 59: 591-592.

14. MATSUMOTO I, FURUSATO M, INOMATA I, WADA T, AIZAWA S. Prostatic cancer presenting as metastatic adenocarcinoma of sphenoid sinus. Acta Pathol Jpn 1986; 36: 1753-1756.

15. RIOJA LA, SANZ VÉLEZ JI, GIL SANZ MJ, ALLEPUZ LOSA C.: Carcinoma de próstata. En: Tratado de Urología. Editado por JR Prous. Jiménez Cruz JF, Rioja Sanz LA. Barcelona 1993; 2: 12051236.

Dr. M.A. Bonillo García

C/ Maestro Buenaventura Pascual, 7 - pta. 8 46020 Valencia

(Trabajo recibido el 25 junio de 2002) 